\title{
Does inflammation precede tau aggregation in early Alzheimer's disease? A PET study
}

DOI:

10.1016/j.nbd.2018.06.004

\section{Document Version}

Accepted author manuscript

Link to publication record in Manchester Research Explorer

\section{Citation for published version (APA):}

Parbo, P., Ismail, R., Sommerauer, M., Stokholm, M. G., Hansen, A. K., Hansen, K. V., Amidi, A., Schaldemose, J. L., Gottrup, H., Braendgaard, H., Eskildsen, S. F., Borghammer, P., Hinz, R., Aanerud, J., \& Brooks, D. J. (2018). Does inflammation precede tau aggregation in early Alzheimer's disease? A PET study. Neurobiology of disease, 117. https://doi.org/10.1016/j.nbd.2018.06.004

\section{Published in:}

Neurobiology of disease

\section{Citing this paper}

Please note that where the full-text provided on Manchester Research Explorer is the Author Accepted Manuscript or Proof version this may differ from the final Published version. If citing, it is advised that you check and use the publisher's definitive version.

\section{General rights}

Copyright and moral rights for the publications made accessible in the Research Explorer are retained by the authors and/or other copyright owners and it is a condition of accessing publications that users recognise and abide by the legal requirements associated with these rights.

\section{Takedown policy}

If you believe that this document breaches copyright please refer to the University of Manchester's Takedown Procedures [http://man.ac.uk/04Y6Bo] or contact uml.scholarlycommunications@manchester.ac.uk providing relevant details, so we can investigate your claim.

\section{OPEN ACCESS}


Does inflammation precede tau aggregation in early Alzheimer's disease?

A PET Study.

\author{
Peter Parbo, ${ }^{1 *}$ Rola Ismail, ${ }^{1}$ Michael Sommerauer, ${ }^{1,2}$ Morten G. Stokholm, ${ }^{1}$ \\ Allan K. Hansen, ${ }^{1}$ Kim V. Hansen, ${ }^{1}$ Ali Amidi, ${ }^{3}$ Jeppe L. Schaldemose ${ }^{1}$, \\ Hanne Gottrup, ${ }^{4}$ Hans Brændgaard, ${ }^{4}$ Simon F. Eskildsen, ${ }^{5}$ Per Borghammer, ${ }^{1}$ \\ Rainer Hinz, ${ }^{6}$ Joel Aanerud, ${ }^{1}$ David J. Brooks ${ }^{1,7}$ \\ ${ }^{1}$ Department of Nuclear Medicine and PET Centre, Aarhus University Hospital, Aarhus, Denmark \\ ${ }^{2}$ Department of Neurology, University of Cologne, Cologne, Germany \\ ${ }^{3}$ Department of Psychology and Behavioural Sciences \& Department of Oncology, \\ Aarhus University \& Aarhus University Hospital, Denmark \\ ${ }^{4}$ Department of Neurology, Aarhus University Hospital, Aarhus, Denmark \\ ${ }^{5}$ Center of Functionally Integrative Neuroscience (CFIN), Aarhus University, Aarhus, Denmark \\ ${ }^{6}$ Wolfson Molecular Imaging Centre, University of Manchester, Manchester, UK \\ ${ }^{7}$ Division of Neuroscience, Newcastle University, Newcastle, UK
}

*Corresponding author:

Peter Parbo, MD, PhD

Department of Nuclear Medicine and PET Centre

Aarhus University Hospital

Nørrebrogade 44, bldg. 10G,

DK-8000 Aarhus, Denmark

E-mail: peter.parbo@clin.au.dk

Running head:

Microglial activation in early Alzheimer's disease.

Number of words in abstract: 213

Number of words in main text: 2808

Number of figures: 4

Number of tables: 1 


\begin{abstract}
:
Objective: Our aim was to assess with positron emission tomography (PET) the temporal and spatial inter-relationships between levels of cortical microglial activation and the aggregated amyloid- $\beta$ and tau load in mild cognitive impairment (MCI) and early Alzheimer's disease (AD).

Methods: Six clinically probable $\mathrm{AD}$ and $20 \mathrm{MCI}$ subjects had inflammation $\left({ }^{11} \mathrm{C}-(R)-\mathrm{PK} 11195\right)$, amyloid $\left({ }^{11} \mathrm{C}-\mathrm{PiB}\right)$ and tau $\left({ }^{18} \mathrm{~F}\right.$-flortaucipir) PET, magnetic resonance imaging (MRI) and a neuropsychological assessment. Parametric images of tracer binding were interrogated at a voxel level and by region of interest analyses.

Results: $55 \%$ of MCI and $83 \%$ of AD subjects had a high amyloid- $\beta$ load. We have previously reported that clusters of correlated amyloid and inflammation levels are present in cortex. Here we found no correlation between levels of inflammation $\left({ }^{11} \mathrm{C}-(R)-\mathrm{PK} 11195 \mathrm{BP}_{\mathrm{ND}}\right)$ and tau $\left({ }^{18} \mathrm{~F}\right.$ flortaucipir SUVR) or MMSE scores in high amyloid- $\beta$ cases.

Interpretation: While correlated levels of amyloid- $\beta$ and inflammation can be seen in MCI, we did not detect an association between levels of cortical tau tangles and inflammation in our series of high amyloid- $\beta$ cases. High levels of inflammation could be seen in amyloid- $\beta$ positive MCI cases where ${ }^{18}$ F-flortaucipir signals were low suggesting microglial activation precedes tau tangle formation. Inflammation levels were higher in high amyloid- $\beta$ MCI than in early AD cases, compatible with it initially playing a protective role.
\end{abstract}

Keywords: Alzheimer's disease; Positron emission tomography; Microglial activation; Amyloid PET; Tau PET.

\title{
Abbreviations:
}

$\mathrm{A} \beta=$ Amyloid- $\beta$; MMSE = Mini-Mental State Examination; PiB = Pittsburgh compound $\mathrm{B}$; PK11195 = ${ }^{11} \mathrm{C}-(R)-\mathrm{PK} 11195 ;$ flortaucipir $={ }^{18}$ F-flortaucipir; $\mathrm{SPM}=$ Statistical parametric mapping; BPM = Biological parametric mapping; FWE = Family-wise error; SUVR = Standardised uptake value ratio; $\mathrm{BP}_{\mathrm{ND}}=$ Binding potential nondisplaceable; $\mathrm{ROI}=$ Region of interest. 


\section{INTRODUCTION}

In Alzheimer's disease, the amyloid cascade hypothesis proposes that deposition of amyloid- $\beta$ (A $\beta$ ) fibrils precedes neurofibrillary tau tangle formation, which in turn leads to neuronal loss and cognitive deficits.[1] Neuroinflammation in the form of microglial and astrocyte activation has been recognised to be a component of this cascade.[2,3] Microglia may express a reparative phenotype, acting to clear cellular debris and remodel synapses or, alternatively, a cidal phenotype releasing cytokines which damage neurons.[4] It remains unresolved which phenotype is preferentially expressed at different time points along the Alzheimer's disease trajectory. Some TSPO positron emission tomography (PET) studies in humans have shown higher signals in prodromal Alzheimer's disease which could support an initially protective role of microglia.[5,30] Soluble A $\beta$ oligomers have been reported to activate microglial cells in cell culture via their Toll-like receptors resulting in neuronal toxicity.[6] Additionally, studies with animal models have observed that the activation of microglia can precede tau aggregation[7] and that cytokines released by microglial cells promote tau hyperphosphorylation and formation of neurofibrillary tangles.[8] Spangenberg et al. have reported that elimination of activated microglia in Alzheimer transgenic mice prevented neuronal and synaptic loss and led to improved memory function in the absence of any change in their $A \beta$ load.[9] These findings suggest a cascade where first $A \beta$ oligomers and fibrils activate microglial cells which in turn release cytokines promoting tau tangle formation.

A majority of subjects with mild cognitive impairment (MCI) are in a transition state between normal aging and dementia and represent prodromal Alzheimer's disease.[10,11] These cases make it possible to investigate the early temporal and spatial inter-relationships between the pathological components of Alzheimer's disease.

The mitochondria of activated microglia express translocator protein $18 \mathrm{kDa}$ (TSPO) allowing these cells to be visualised in vivo with PET ligands for this protein. The TSPO marker ${ }^{11} \mathrm{C}-(R)-\mathrm{PK} 11195$ PET detects increased levels of microglial activation as an early event in Alzheimer's disease.[12,13] More recently, in vivo tau tangle imaging with PET has become available with the pyrido-indole ${ }^{18}$ F-flortaucipir (also know as AV-1451 or T807). Studies have reported increased cortical tau aggregation in vivo in MCI, Alzheimer's disease[14,15] and occasionally in healthy elderly subjects.[16]

The aim of this current cross-sectional study was to investigate the inter-relationships between the components of early Alzheimer's disease pathophysiology. We have scanned MCI subjects with a raised amyloid load at high risk of developing Alzheimer's disease and cases with early 
Alzheimer's dementia. We used multiple PET tracers to examine the relationship between A $\beta$ deposition, microglial activation and tau tangle aggregation.

\section{METHODS}

\section{Study subjects}

All cognitively impaired subjects were recruited from memory clinics or by advertisement and they were assessed as previously described.[13] Initially, our ${ }^{11} \mathrm{C}-\mathrm{PiB}$ (PiB) and ${ }^{11} \mathrm{C}-(R)-\mathrm{PK} 11195$ (PK11195) PET program was designed to investigate the temporal and spatial relationships between $\mathrm{A} \beta$ fibril load and levels of inflammation in MCI. During the conduct of the study ${ }^{18} \mathrm{~F}$ flortaucipir (flortaucipir) PET became available to determine levels and distribution of tau aggregation. Twenty of our $42 \mathrm{MCI}$ cases agreed to have scans with all three PET tracers within a reasonable time window of 10 weeks. We present cross-sectional data from these $20 \mathrm{MCI}$ and an additional six cases with early Alzheimer's disease.

Our Alzheimer's disease patients were recruited from the local dementia clinic, where experienced neurologists diagnosed the patients according to ICD-10 clinical criteria. MCI patients fulfilled the Petersen criteria.[10] The age of Alzheimer's disease patients and MCI subjects ranged between 5085 years. They had a modified Hachinski Ischemic Scale score $\leq 4$, and a Geriatric Depression Scale (version with 15 questions) score $\leq 6$. None had a neurological or psychiatric disorder, were taking drugs associated with cognitive impairment or had any contraindication for magnetic resonance imaging (MRI). All MCI and Alzheimer's disease subjects were assessed with a neuropsychological test battery comprised of standardized neuropsychological tests assessing memory, language, visuospatial function, processing speed and executive function as described previously.[13]

Additional flortaucipir[17] and PK11195[18] PET scans of healthy controls were available from an in-house database which we combined with our own control data, providing a total of 22 flortaucipir, $20 \mathrm{PK} 11195$, and $12 \mathrm{PiB}$ healthy controls (HC). Seven of the controls had both PK11195 and PiB PET. The pooled HC subjects were recruited via advertisement, assessed with neurological and cognitive screening (Mini-Mental State Examination (MMSE) scores), and a depression scale (either the Geriatric Depression Scale or the Major Depression Index from ICD10). All subjects were scanned using the same HRRT PET scanner and identical scanning protocols. 
The Central Denmark Region Committees on Health Research Ethics approved the study in accordance with the declaration of Helsinki. All participants signed an informed written consent prior to enrolment in the study.

\section{Image processing}

MRI and PET were acquired as previously described.[13] MRI was performed with a Skyra 3 Tesla system (Siemens, Erlangen, Germany), and PET scans were acquired with a High Resolution Research Tomograph (ECAT HRRT; CTI/Siemens, Knoxville, TN, USA). A T1 MP2RAGE (Magnetization Prepared Rapid Gradient-Echo with two gradient echo images) sequence was used for co-registration of MRI with PET, normalisation of images into standard MNI space, and generation of grey matter masks. MINC software (http://en.wikibooks.org/wiki/MINC)[19] was used for segmenting MRI volumes into grey (GM) and white (WM) matter images and cerebrospinal fluid (CSF),[20] and to spatially normalise the MRI and PET images.[21] The GM masks were convolved with a probabilistic atlas[22] to "individualise" subject regions of interest (ROIs) to their GM. A composite ROI for measuring global cortical flortaucipir SUVR was generated as a volume-weighted average of temporal, parietal and occipital regions.[23]

Average doses of $410 \pm 34 \mathrm{MBq}{ }^{11} \mathrm{C}-\mathrm{PiB}$ (PiB; Pittsburgh compound $\mathrm{B}$ )[24] and $358 \pm 35 \mathrm{MBq}$

${ }^{18} \mathrm{~F}$-flortaucipir were administered as intravenous boluses. PiB PET was acquired for 50 minutes in list mode 40-90 minutes post injection. Flortaucipir PET was acquired for 40 minutes in list mode 80-120 minutes post injection. The spatially normalised PiB and flortaucipir images were summed from 60-90 and 80-100 minutes, respectively, and voxel signal was divided by the mean signal from the individual's cerebellar crus GM to generate PiB and flortaucipir standardised uptake value ratio (SUVR) images.

An average dose of $406 \pm 33 \mathrm{MBq}{ }^{11} \mathrm{C}-(R)-\mathrm{PK} 11195$ was injected intravenously over 10 seconds, followed by a $10 \mathrm{~mL}$ saline flush. Emission scans were initiated with a 30 second "background" frame before injection of PK11195. The total dynamic scan time was 60.5 minutes (list mode). Frames were re-binned as: 1x 30 seconds 'background', 6x 10s, 2x 30s, 2x 60s, 3x 120s, 10x 300s. PK11195 binding potential $\left(\mathrm{BP}_{\mathrm{ND}}\right)$ maps were generated at a voxel level using the Simplified Reference Tissue Model[25] implemented in MATLAB. Supervised Cluster Analysis with 6classes[26] was applied to the dynamic images to localise a cluster of voxels which provided a reference tissue input function representing normal grey matter uptake kinetics. However, these non-specific uptake reference clusters can contain occasional voxels with low specific signal and this can result in apparent negative binding potentials in areas of low tracer signal. 


\section{High and low A $\beta$ load}

All our MCI cases who showed visual evidence on a blinded read of cortical amyloid had a composite cortical PiB SUVR of 1.7 or higher (Fig. 1A). The composite cortical PiB ROI sampled frontal, middle/inferior temporal, lateral parietal, precuneus and posterior cingulate cortical areas. Rather than label individuals as $A \beta$ positive or negative we prefer to use the terms high and low $A \beta$ load given that Thal et al.[27] have reported that conventional thresholds used to detect raised ${ }^{18} \mathrm{~F}$ flutemetamol ( $\left.{ }^{18} \mathrm{~F}-\mathrm{PiB}\right)$ PET can miss early (stage 1 and 2) $\mathrm{A} \beta$ deposition later found at postmortem.

\section{Statistical and Biological Parametric Mapping}

Statistical Parametric Mapping (SPM8; Wellcome Trust Centre for Neuroimaging) was used to perform between-group comparisons (two-sample t-test) of tracer uptake and correlation analyses (multiple regression) between MMSE scores and tracer uptake at a voxel level. Biological Parametric Mapping (BPM) (using the BPM toolbox in SPM5)[28] was used to localise voxels where individual levels of flortaucipir SUVR and PK11195 BP $\mathrm{ND}_{\mathrm{ND}}$, and flortaucipir SUVR and PiB SUVR were correlated. Parametric maps of flortaucipir SUVR, PiB SUVR, and PK11195 BP were smoothed to a total of $8 \mathrm{~mm}$ full width at half maximum prior to voxel level analyses. An averaged cortical GM mask (without cerebellum) was used for explicit masking within the SPM and BPM software. Voxel analysis of flortaucipir and PiB SUVR images were thresholded at $P<$ 0.001 uncorrected. Our figures only display clusters that survived a family-wise error (FWE) cluster-level correction at $P<0.05$.

\section{Statistical analysis}

STATA 13.1 (StataCorp LP, Texas, USA) and Prism 6 (GraphPad Software, La Jolla, CA, USA) were used for statistical analyses and graphical displays. Data was assessed for normality using the Shapiro-Wilk normality test, and by plotting data in histograms and Q-Q plots. Table data were compared with one-way ANOVAs and post hoc uncorrected pairwise group comparisons were used for normally distributed continuous variables, Fisher's exact tests for nominal variables and Kruskal-Wallis test with post hoc uncorrected Wilcoxon rank-sum test for ordinal variables as appropriate. Spearman's rank sum test was applied for ROI correlation analyses. Repeated measures two-way ANOVA model with three groups ( $\mathrm{HC}$, high $\mathrm{A} \beta \mathrm{MCI}$ and high $\mathrm{A} \beta \mathrm{AD}$ ) and eight regions was used for analysis of PK111195 $\mathrm{BP}_{\mathrm{ND}}$ levels. $P$ values below 0.05 were considered 
statistical significant. Neuropsychological data were transformed into z-scores using normative data from our healthy controls who were also assessed with the neuropsychological test battery. Subsequently, domain-specific z-scores were calculated as the mean of domain-relevant tests.

\section{RESULTS}

Five $(83 \%)$ of the six clinically probable Alzheimer's disease subjects were categorised as having a high $\mathrm{A} \beta$ load, while our $20 \mathrm{MCI}$ cases were divided into 11 (55\%) with high $\mathrm{A} \beta$ and nine (45\%) with low $\mathrm{A} \beta$ loads (Fig. 1A; see table 1 for an overview on characterisation of $\mathrm{HC}$ and low/high $\mathrm{A} \beta$ $\mathrm{MCI} / \mathrm{AD})$. Additionally, figure 1A reveals two $\mathrm{HCs}(17 \%)$ with visual and quantitative high $\mathrm{A} \beta$ load and one AD subject with visual and quantitative low $A \beta$ load. The combined group of 16 high $\mathrm{A} \beta \mathrm{MCI} / \mathrm{AD}$ cases showed significantly higher levels of composite flortaucipir SUVR compared to HC (mean $1.53 \pm 0.39$ vs. mean $1.19 \pm 0.09 ; P=0.0005)$ (Fig. 1B). We found no PET evidence that our MCI subjects with low PiB had a tauopathy.

Figure 2 presents surface SPMs of the high A $\beta$ MCI/AD group which showed raised flortaucipir signal with a posterior emphasis when compared to $\mathrm{HC}$ and low $\mathrm{A} \beta \mathrm{MCI} / \mathrm{AD}$. This posterior pattern targeted parietal, precuneus, posterior cingulate, lateral and medial temporal cortices, in line with the distribution reported by Johnson et al.[29] except that our high A $\beta$ MCI/AD sample displayed less tau in their occipital cortex. The high $\mathrm{A} \beta \mathrm{MCI} / \mathrm{AD}$ group had significantly reduced processing speed, visuospatial function, global memory, and executive performance compared to controls and low $\mathrm{A} \beta \mathrm{MCI} / \mathrm{AD}$ subjects (table 1).

A group effect was found when assessing the levels of PK11195 binding in subgroups of HC, high $\mathrm{A} \beta \mathrm{MCI}$ and $\mathrm{AD}$ subjects $(\mathrm{F}(2,33)=3.57 ; P=0.039)$ (Fig. 3). Post hoc group comparisons (uncorrected) revealed higher levels of PK11195 $\mathrm{BP}_{\mathrm{ND}}$ in the high $\mathrm{A} \beta \mathrm{MCI}$ group compared to $\mathrm{HC}$ subjects in all cortical regions bar the lateral parietal area. Compared to the AD subjects the high $\mathrm{A} \beta$ MCI group showed raised PK11195 $\mathrm{BP}_{\mathrm{ND}}$ in the posterior cingulate $(P=0.015)$ and lateral temporal $(P=0.036)$ regions. There were no significant differences in inflammation levels across regions between $\mathrm{HC}$ and $\mathrm{AD}$ subjects but there were only $5 \mathrm{AD}$ cases providing low power.

\section{Correlations between levels of activated microglia, $\mathrm{A} \beta$ and tau}

We previously reported clusters of correlated amyloid load and inflammation in the cortex of high $\mathrm{A} \beta$ MCI cases.[13] In the present group of 16 high A $\beta$ MCI/AD subjects the BPM of PiB SUVR 
and PK11195 $\mathrm{BP}_{\mathrm{ND}}$ also detected clusters of correlated amyloid load and inflammation in parietal, lateral temporal and frontal areas, but at a lower threshold $(\mathrm{p}<0.05$ with an extent threshold of 100 voxels). BPM interrogation of flortaucipir SUVR and PK11195 $\mathrm{BP}_{\mathrm{ND}}$ at a voxel level did not reveal any significant clusters of positive correlations between tau and inflammation levels. In support of this, correlation analysis within frontal, hippocampal, parahippocampal, lateral and posterior temporal, precuneus, posterior cingulate and lateral parietal ROIs also did not reveal any

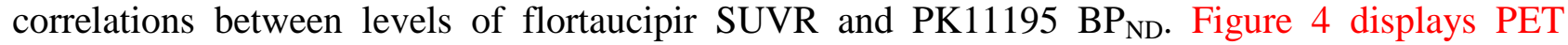
images of one high $\mathrm{A} \beta$ case with relatively increased levels of PK11195 binding but low levels of flortaucipir SUVR, and one high A $\beta$ case with low PK11195 binding and high flortaucipir SUVR. We found no significant correlation between levels of inflammation $\left(\mathrm{PK} 11195 \mathrm{BP}_{\mathrm{ND}}\right)$ and MMSE scores, using ROI ( $P$-values between 0.31 and 0.93 ) or voxel-level analyses.

\section{DISCUSSION}

This PET study assessed the in vivo inter-relationships between levels and extent of microglial activation, cortical $A \beta$ plaque and tau tangle load in the prodromal/early phases of Alzheimer's disease. While we previously reported that clusters of correlated levels of microglial activation and $\mathrm{A} \beta$ deposition in cortical association areas were present in MCI with raised $\mathrm{A} \beta$, we did not detect any significant association between tau load and inflammation levels in this series. This may simply be a sensitivity problem reflecting a lower density of tangles compared to $A \beta$ plaques and the low specific binding signal provided by PK11195 PET. However, as PET can clearly detect the presence of both tau and inflammation in the posterior cortical areas of MCI/early Alzheimer's disease cases with raised $A \beta$, the lack of correlation of their levels may reflect a true situation microglial activation and tau aggregation occurring via independent mechanisms with different trajectories. Inflammation may well be an earlier phenomenon linked to A $\beta$ deposition at the onset of disease, the activated microglial cells trying to restore brain function by engulfing and clearing $\mathrm{A} \beta$ oligomers/fibrils. While initially raised inflammation acts as a protective mechanism to try and clear $\mathrm{A} \beta$, this fails and activated microglia decline. Around this time tau phosphorylation and aggregation begins.[30] Our finding of increased inflammation levels in high amyloid MCI (Fig. 3) is in line with Hamelin et al.[5] A study from Carter and colleagues has reported higher cortical 11C-DED binding from astrocytes in $\mathrm{PiB}+\mathrm{MCI}$ compared to both controls and $\mathrm{AD}$ subjects 
suggesting that astrocytes may also be activated in early $\mathrm{AD}$, levels later falling.[31] Given the differing temporal trajectories of amyloid plaque formation, inflammation, and tau aggregation, it is perhaps not surprising to find no regional correlation between PK11195 and flortaucipir uptake across our 16 high $\mathrm{A} \beta \mathrm{MCI} / \mathrm{AD}$ subjects.

We did not find significant correlations at a voxel level between MMSE scores and PK11195 BP $\mathrm{ND}_{\mathrm{N}}$ in our 16 high $\mathrm{A} \beta \mathrm{MCI} / \mathrm{AD}$ subjects. The exact trajectory of microglial activation in AD is still not clear but PK11195 signal can range from high to near normal in early MCI. As AD progresses and tau rises there may then be a second wave of cidal inflammation. This biphasic time course could explain a failure to find regional correlations between levels of cognition and PK11195 signal.

As our study is cross-sectional and comprised mainly MCI cases, we cannot delineate the trajectory of Alzheimer's disease from these data. Although the current study is based on a small sample size of participants, the findings show microglial activation in high A $\beta$ MCI cases who have not, but are likely to, develop cortical tau aggregations. A limitation of this study is that our control subjects were not scanned with all three PET tracers; this was due to dosimetry regulations when administering radioactive materials to healthy control subjects.

Compared to newer TSPO tracers now available, PK11195 has a relatively low signal-to-noise ratio and this could reduce our power to identify correlations with inflammation. On the other hand, PK11195 binding is not significantly influenced by the TSPO polymorphism simplifying the interpretation of findings.[33] Specific PK11195 uptake is modelled using a tissue reference cluster of voxels representing the kinetics of normal cortical grey matter. However, the non-specific uptake reference cluster identified by discriminant analysis can contain occasional voxels with some specific signal and produces apparent negative binding potentials in some regions where tracer brain uptake is low in MCI.

One of our clinically diagnosed Alzheimer's disease cases was categorised by PET as having low A $\beta$. This could possibly be due to a limited sensitivity of PiB PET for detecting amyloid but is more likely to reflect an incorrect diagnosis and implicate other pathologies, such as small vessel disease, as a cause of the cognitive impairment.[34]

In summary, we have investigated the inter-relationship between levels of microglial activation and the aggregation load of $A \beta$ and tau protein in a group of prodromal and early Alzheimer's disease cases. While we previously reported clusters of correlated $\mathrm{A} \beta$ and inflammation levels in association cortical areas of our prodromal AD cases, we failed to find a correlation between tau and inflammation levels in this series. As amyloid and inflammation can be present in the absence 
of tau tangles in MCI, it seems likely that microglia activation precedes tau tangle formation in the cascade of AD pathology.

\section{Acknowledgements}

This study was financially supported by grants from the Danish Council of Independent Research [grant no. DFF-1331-00184], AUFF Neurodin (Aarhus University) and the Lundbeck Foundation [grant no. R140-2013-13245]. We thank Avid Radiopharmaceuticals, Inc., for providing precursor for the ${ }^{18} \mathrm{~F}$-flortaucipir tracer synthesis.

\section{Financial disclosures}

Dr. Brooks reports grants from Danish Council of Independent Research and the Lundbeck Foundation in support of this study and from the EU FP7 programme; lecturing fees and nonfinancial support from GE Healthcare and Isotopia outside the submitted work; Dr. Hinz reports grants from European Commission, during the conduct of the study; all other authors have nothing to disclose. 


\section{REFERENCES}

1 Hardy J, Higgins G. Alzheimer's disease: the amyloid cascade hypothesis. Science (80- ) 1992;256:184-5. doi:10.1126/science. 1566067

2 Meda L, Cassatella MA, Szendrei GI, et al. Activation of microglial cells by beta-amyloid protein and interferon-gamma. Nature. 1995;374:647-50. doi:10.1038/374647a0

3 Fan Z, Aman Y, Ahmed I, et al. Influence of microglial activation on neuronal function in Alzheimer's and Parkinson's disease dementia. Alzheimer's Dement 2015;11:608-621.e7. doi:10.1016/j.jalz.2014.06.016

4 Varnum MM, Ikezu T. The Classification of Microglial Activation Phenotypes on Neurodegeneration and Regeneration in Alzheimer's Disease Brain. Arch Immunol Ther Exp (Warsz) 2012;60:251-66. doi:10.1007/s00005-012-0181-2

5 Hamelin L, Lagarde J, Dorothée G, et al. Early and protective microglial activation in Alzheimer's disease: A prospective study using 18F-DPA-714 PET imaging. Brain 2016;139:1252-64. doi:10.1093/brain/aww017

6 Heneka MT, Carson MJ, Khoury J El, et al. Neuroinflammation in Alzheimer's disease. Lancet Neurol 2015;14:388-405. doi:10.1016/S1474-4422(15)70016-5

7 Yoshiyama Y, Higuchi M, Zhang B, et al. Synapse Loss and Microglial Activation Precede Tangles in a P301S Tauopathy Mouse Model. Neuron 2007;53:337-51. doi:10.1016/j.neuron.2007.01.010

8 Maphis N, Xu G, Kokiko-Cochran ON, et al. Reactive microglia drive tau pathology and contribute to the spreading of pathological tau in the brain. Brain 2015;138:1738-55. doi:10.1093/brain/awv081

9 Spangenberg EE, Lee RJ, Najafi AR, et al. Eliminating microglia in Alzheimer's mice prevents neuronal loss without modulating amyloid- $\beta$ pathology. Brain 2016;139:1265-81. doi:10.1093/brain/aww016

10 Petersen RC, Doody R, Kurz A, et al. Current Concepts in Mild Cognitive Impairment. Arch Neurol 2001;58:1985-92.

11 Dubois B, Albert ML. Amnestic MCI or prodromal Alzheimer's disease? Lancet 2004;3:246-8. doi:10.1087/095315104322710287

12 Cagnin A, Brooks DJ, Kennedy AM, et al. In-vivo measurement of activated microglia in dementia. Lancet 2001;358:461-7. doi:10.1016/S0140-6736(01)05625-2

13 Parbo P, Ismail R, Hansen K V, et al. Brain inflammation accompanies amyloid in the majority of mild cognitive impairment cases due to Alzheimer's disease. Brain 2017;140:2002-11. doi:10.1093/brain/awx120

14 Chien DT, Bahri S, Szardenings AK, et al. Early Clinical PET Imaging Results with the Novel PHF-Tau Radioligand [F-18]-T807. J Alzheimer's Dis 2013;34:457-68. doi:10.3233/JAD-122059

15 Ossenkoppele R, Schonhaut DR, Schöll M, et al. Tau PET patterns mirror clinical and neuroanatomical variability in Alzheimer's disease. Brain 2016;139:1551-67. doi:10.1093/brain/aww027

16 Schöll M, Lockhart SN, Schonhaut DR, et al. PET Imaging of Tau Deposition in the Aging Human Brain. Neuron 2016;89:971-82. doi:10.1016/j.neuron.2016.01.028

17 Hansen AK, Knudsen K, Lillethorup TP, et al. In vivo imaging of neuromelanin in Parkinson's disease using 18F-AV-1451 PET. Brain 2016;139:2039-49. doi:10.1093/brain/aww098

18 Stokholm MG, Iranzo A, Østergaard K, et al. Assessment of neuroinflammation in patients with idiopathic rapid-eye-movement sleep behaviour disorder: a case-control study. Lancet Neurol 2017;4422:1-8. doi:10.1016/S1474-4422(17)30173-4 
19 Vincent RD, Neelin P, Khalili-Mahani N, et al. MINC 2.0: A Flexible Format for MultiModal Images. Front Neuroinform 2016;10:0-8. doi:10.3389/fninf.2016.00035

20 Zijdenbos AP, Dawant BM, Margolin RA, et al. Morphometric analysis of white matter lesions in MR images: method and validation. IEEE Trans Med Imaging 1994;13:716-24. doi:10.1109/42.363096

21 Collins DL, Neelin P, Peters TM, et al. Automatic 3D intersuject Registration fo MR Volumetric Data in Standardized Talairach Space. J. Comput. Assist. Tomogr. 1994;18:192205. doi:10.1093/cercor/10.4.433

22 Hammers A, Allom R, Koepp MJ, et al. Three-dimensional maximum probability atlas of the human brain, with particular reference to the temporal lobe. Hum Brain Mapp 2003;19:22447. doi:10.1002/hbm.10123

23 Pontecorvo MJ, Devous MD, Navitsky M, et al. Relationships between flortaucipir PET tau binding and amyloid burden, clinical diagnosis, age and cognition. Brain 2017;:aww334. doi:10.1093/brain/aww334

24 Klunk WE, Engler H, Nordberg A, et al. Imaging brain amyloid in Alzheimer's disease with Pittsburgh Compound-B. Ann Neurol 2004;55:306-19. doi:10.1002/ana.20009

25 Lammertsma AA, Hume SP. Simplified Reference Tissue Model for PET Receptor Studies. Neuroimage 1996;4:153-8. doi:10.1006/nimg.1996.0066

26 Turkheimer FE, Edison P, Pavese N, et al. Reference and target region modeling of [11C](R)-PK11195 brain studies. J Nucl Med 2007;48:158-67. doi:48/1/158 [pii]

27 Thal DR, Beach TG, Zanette M, et al. $\left[{ }^{18} \mathrm{~F}\right]$ flutemetamol amyloid positron emission tomography in preclinical and symptomatic Alzheimer's disease: Specific detection of advanced phases of amyloid- $\beta$ pathology. Alzheimer's Dement 2015;11:975-85. doi:10.1016/j.jalz.2015.05.018

28 Casanova R, Srikanth R, Baer A, et al. Biological parametric mapping: A statistical toolbox for multimodality brain image analysis. Neuroimage 2007;34:137-43. doi:10.1016/j.neuroimage.2006.09.011

29 Johnson KA, Schultz A, Betensky RA, et al. Tau positron emission tomographic imaging in aging and early Alzheimer disease. Ann Neurol 2016;79:110-9. doi:10.1002/ana.24546

30 Fan Z, Brooks DJ, Okello A, et al. An early and late peak in microglial activation in Alzheimer's disease trajectory. Brain 2017;:aww349. doi:10.1093/brain/aww349

31 Carter SF, Scholl M, Almkvist O, et al. Evidence for Astrocytosis in Prodromal Alzheimer Disease Provided by 11C-Deuterium-L-Deprenyl: A Multitracer PET Paradigm Combining 11C-Pittsburgh Compound B and 18F-FDG. J Nucl Med 2012;53:37-46. doi:10.2967/jnumed.110.087031

32 Cho H, Choi JY, Lee SH, et al. Excessive tau accumulation in the parieto-occipital cortex characterizes early-onset Alzheimer's disease. Neurobiol Aging 2017;53:103-11. doi:10.1016/j.neurobiolaging.2017.01.024

33 Owen DR, Howell OW, Tang S-P, et al. Two binding sites for [3H]PBR28 in human brain: implications for TSPO PET imaging of neuroinflammation. J Cereb Blood Flow Metab 2010;30:1608-18. doi:10.1038/jcbfm.2010.63

34 Kapasi A, DeCarli C, Schneider JA. Impact of multiple pathologies on the threshold for clinically overt dementia. Acta Neuropathol 2017;134:171-86. doi:10.1007/s00401-0171717-7 


\section{FIGURE LEGENDS}

Figure 1. Dotplots of composite ${ }^{11} \mathrm{C}-\mathrm{PiB}$ and ${ }^{18} \mathrm{~F}$-flortaucipir SUVR.

A: Composite PiB SUVR in HC, MCI and AD subjects. Dashed red squares marks the 16 high A $\beta$ MCI/AD subjects used in subsequent analyses; Group means are marked by +++ and horizontal dashed black lines marks \pm 1 SD. B: Composite flortaucipir SUVR in HC, low A $\beta \mathrm{MCI} / \mathrm{AD}$ and high $\mathrm{A} \beta \mathrm{MCI} / \mathrm{AD}$.

Figure 2. Statistical parametric surface maps of significantly raised flortaucipir SUVR in high A $\beta$ MCI/AD subjects $(n=16)$ compared to $\mathrm{HC}(\mathrm{n}=22)$, and compared to low A $\beta$ MCI/AD $(n=10)$. No clusters of raised flortaucipir SUVR were seen when comparing low A $\beta$ MCI/AD vs. HC subjects. T-maps are thresholded at $P<0.001$ uncorrected and FWE cluster-level corrected at $P<$ 0.05 .

Figure 3. Regional levels of PK11195 $\mathrm{BP}_{\mathrm{ND}}$ in $\mathrm{HC}$, high $\mathrm{A} \beta \mathrm{MCI}$ and high $\mathrm{A} \beta \mathrm{AD}$ subjects. Height of bars and error bars mark means with 95\% CI.

Figure 4. PET images of two high $\mathrm{A} \beta \mathrm{MCI}$ subjects.

Individual PiB SUVR, PK11195 $\mathrm{BP}_{\mathrm{ND}}$ and flortaucipir SUVR PET images of two high A $\beta$ MCI subjects. The first case shows raised inflammation but low tau while the second case shows little inflammation but raised tau. 
Table 1. Participant characterisation

\begin{tabular}{|c|c|c|c|c|c|}
\hline & \multicolumn{3}{|c|}{$\mathrm{HC}$} & \multicolumn{2}{|c|}{$\mathrm{MCI} / \mathrm{AD}$} \\
\hline & $\begin{array}{l}{ }^{11} \mathrm{C}-\mathrm{PiB} \\
(\mathrm{n}=12)\end{array}$ & $\begin{array}{l}{ }^{11} \mathrm{C}-\mathrm{PK} 11195 \\
(\mathrm{n}=20)\end{array}$ & $\begin{array}{l}{ }^{18} \text { F-flortaucipir } \\
(n=22)\end{array}$ & $\begin{array}{c}\text { Low } A \beta \\
(n=10)\end{array}$ & $\begin{array}{l}\text { High } A \beta \\
(n=16)\end{array}$ \\
\hline Age, mean [range], y & $69[59-77]$ & $67[58-80]$ & $69[53-84]$ & 65 [50-79] & $72[54-81]$ \\
\hline Female, No. $(\%)$ & $7(58 \%)$ & $8(40 \%)$ & $7(32 \%)$ & $4(40 \%)$ & $6(38 \%)$ \\
\hline Education, mean $\pm \mathrm{SD}$, years & $13 \pm 2.5$ & $14 \pm 2.5$ & $14 \pm 2.5$ & $12 \pm 1.6^{\mathrm{a}}$ & $12 \pm 2.2^{\mathrm{a}}$ \\
\hline Diagnosis of AD, No. (\%) & & & & $1(10 \%)$ & $5(31 \%)$ \\
\hline NSAID use, No. (\%) & $1(8 \%)$ & $1(5 \%)$ & $3(14 \%)$ & $2(20 \%)$ & $4(25 \%)$ \\
\hline Cholinesterase inhibitor use, No. (\%) & $0(0 \%)$ & $0(0 \%)$ & $0(0 \%)$ & $0(0 \%)$ & $5(31 \%)^{\mathrm{a}, \mathrm{b}}$ \\
\hline CDR sum-of-boxes, mean \pm SD & & & & $1.3 \pm 1.1$ & $2.7 \pm 2.1^{\mathrm{a}}$ \\
\hline MMSE score, mean \pm SD & $29 \pm 1.2$ & $29 \pm 1.5$ & $29 \pm 1.4$ & $27 \pm 2.1$ & $25 \pm 3.8^{\mathrm{a}}$ \\
\hline \multicolumn{4}{|c|}{ Domain z-scores from neuropsychological test battery } & $\begin{array}{c}\text { Low } A \beta \\
(n=10)\end{array}$ & $\begin{array}{l}\text { High } A \beta \\
(\mathrm{n}=16)\end{array}$ \\
\hline \multicolumn{4}{|c|}{ Processing speed $z$-score, mean \pm SD } & $-0.36 \pm 0.9$ & $-2.0 \pm 1.0^{\mathrm{b}}$ \\
\hline \multicolumn{4}{|c|}{ Visuospatial function $\mathrm{z}$-score, mean $\pm \mathrm{SD}$} & $-0.04 \pm 1.9$ & $-2.1 \pm 2.6^{b}$ \\
\hline \multicolumn{4}{|c|}{ Global memory z-score, mean \pm SD } & $-0.7 \pm 1.4$ & $-2.1 \pm 1.1^{b}$ \\
\hline \multicolumn{4}{|c|}{ Language $\mathrm{z}$-score, mean $\pm \mathrm{SD}$} & $-0.6 \pm 1.3$ & $-1.5 \pm 1.4$ \\
\hline \multicolumn{4}{|c|}{ Executive function $\mathrm{z}$-score, mean $\pm \mathrm{SD}$} & $-0.3 \pm 1.0$ & $-2.1 \pm 1.1^{b}$ \\
\hline \multicolumn{4}{|c|}{ Global composite score $\mathrm{z}$-score, mean $\pm \mathrm{SD}$} & $-0.4 \pm 1.1$ & $-1.9 \pm 1.0^{\mathrm{b}}$ \\
\hline
\end{tabular}

Abbreviations: $\mathrm{HC}=$ Healthy controls; $\mathrm{MCI}=$ Mild cognitive impairment; $\mathrm{AD}=$ Alzheimer's disease;

$\mathrm{A} \beta=$ amyloid $-\beta ;$ NSAID $=$ Non-steroidal anti-inflammatory drug; $\mathrm{CDR}=$ Clinical Dementia Rating.

Significant differences are marked with: ${ }^{\mathrm{a}} P<0.05$ versus HC; ${ }^{\mathrm{b}} P<0.05$ versus Low A $\beta$. Group differences are tested (using parametric and non-parametric testing where appropriate). Whenever possible, the three controls samples are summarised for statistical comparisons. 


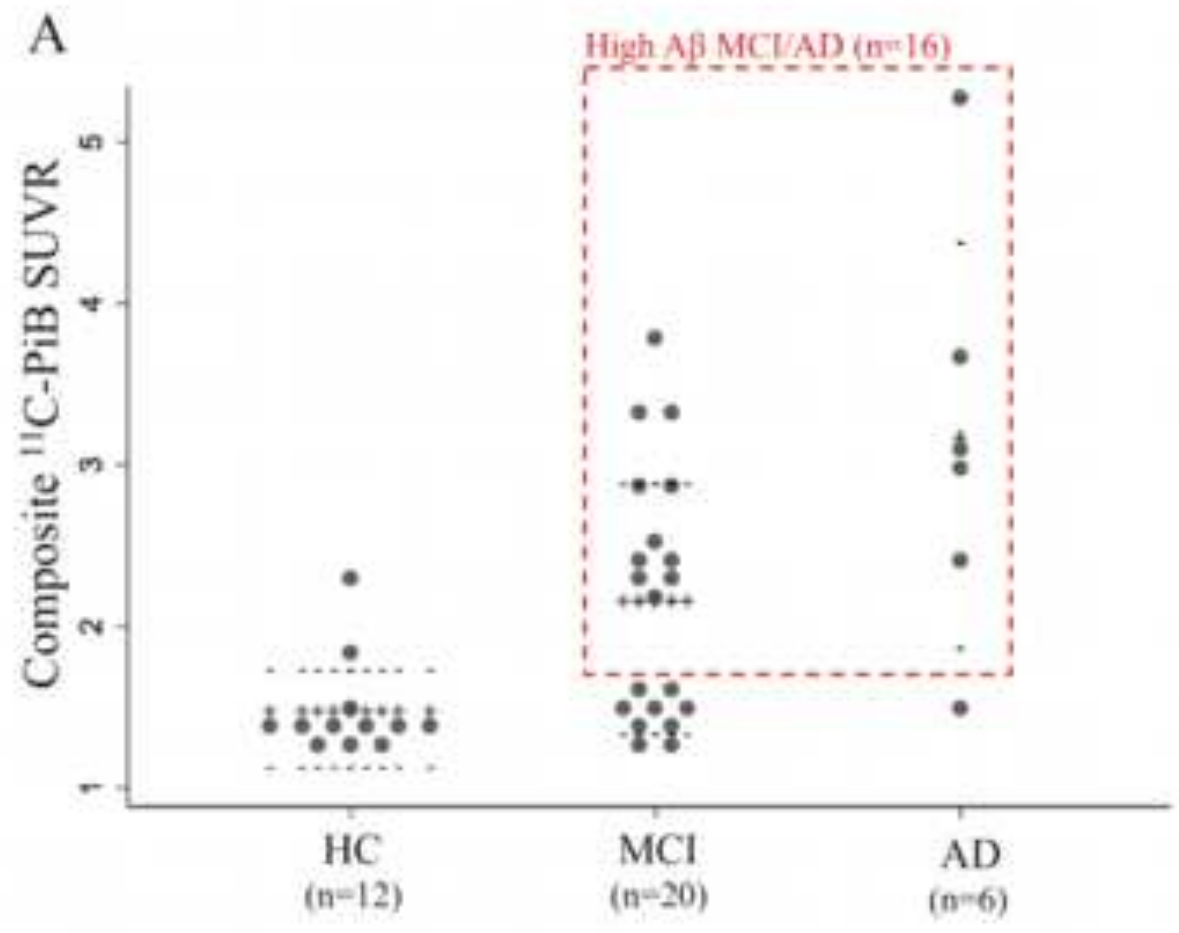

B

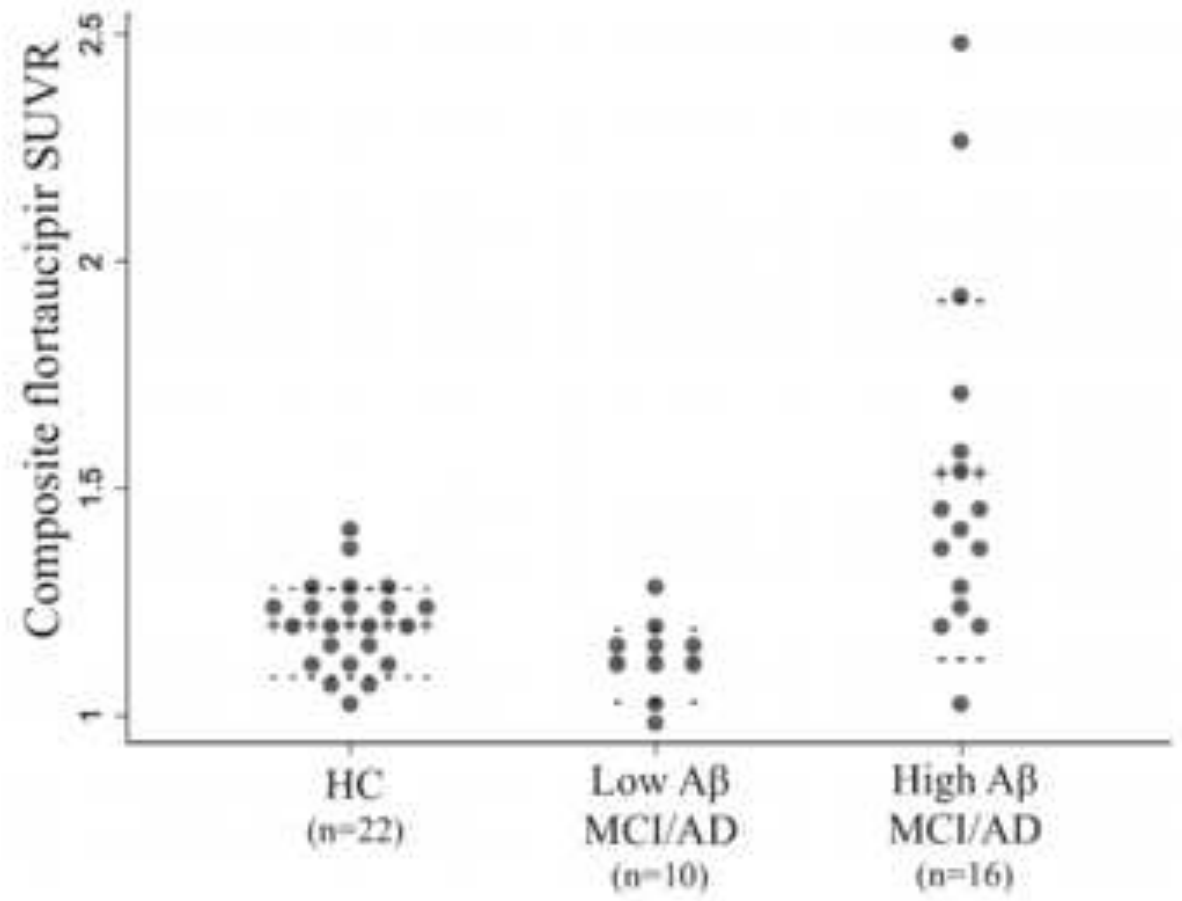


Click here to download high resolution image

\section{Distribution of increased ${ }^{18} \mathrm{~F}$-flortaucipir SUVR}

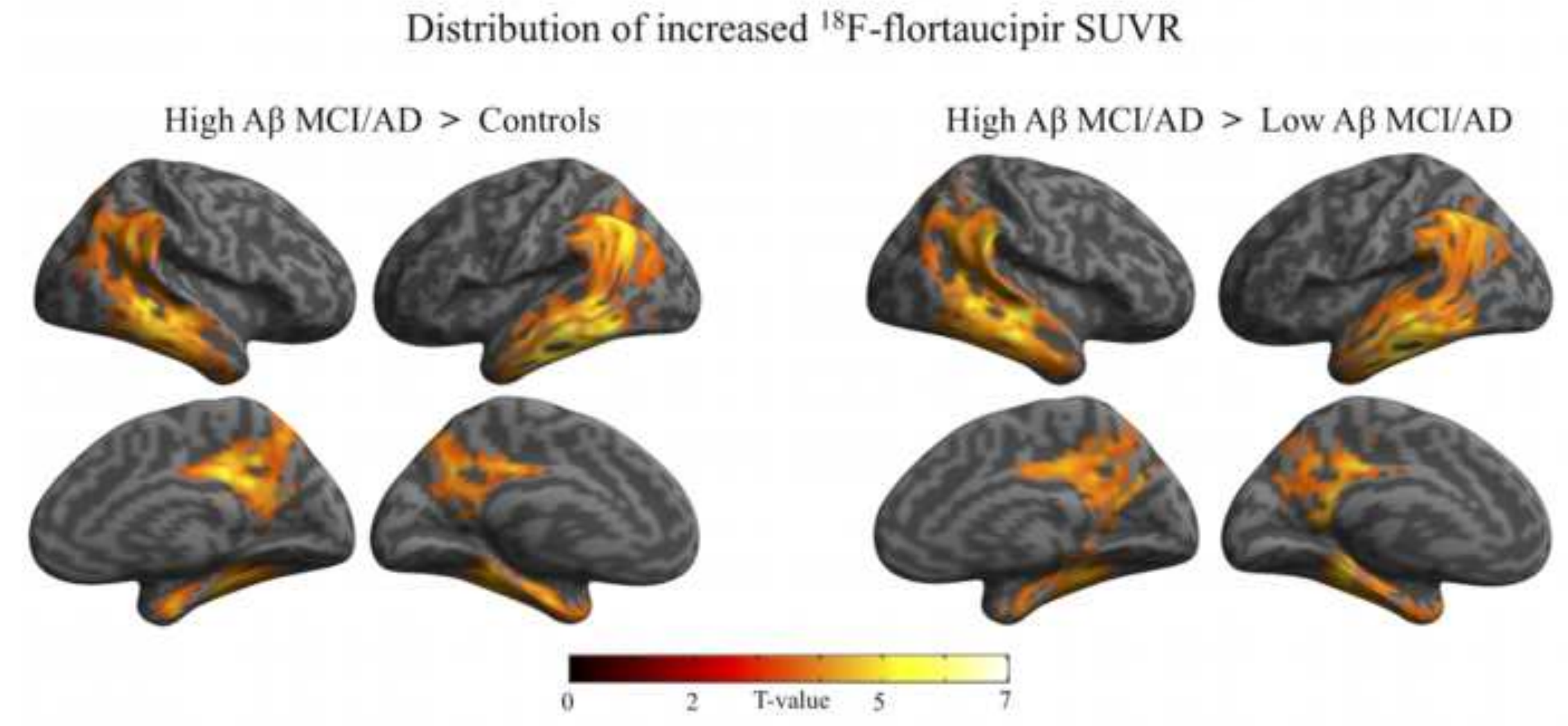




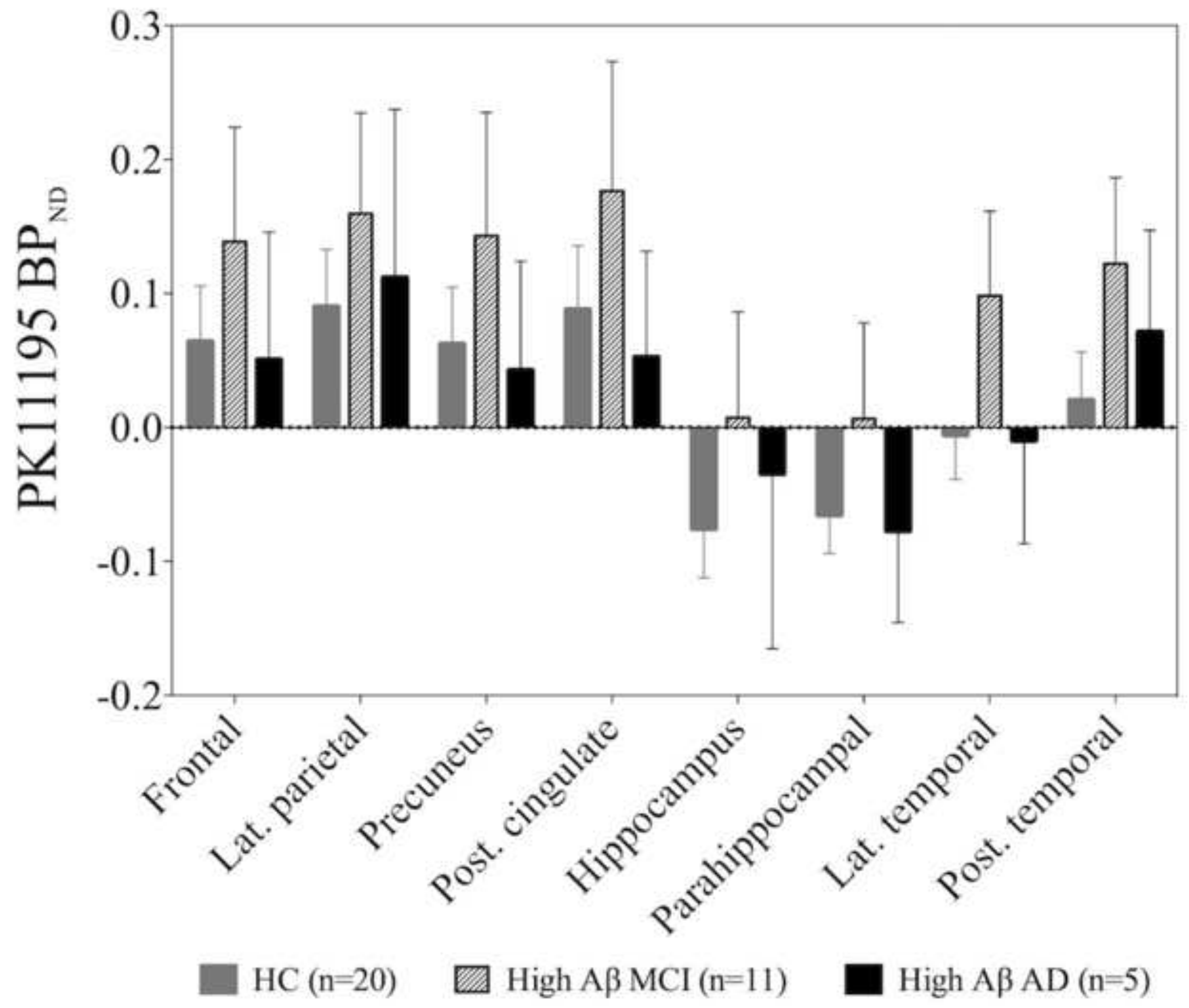




\section{Click here to download high resolution image}

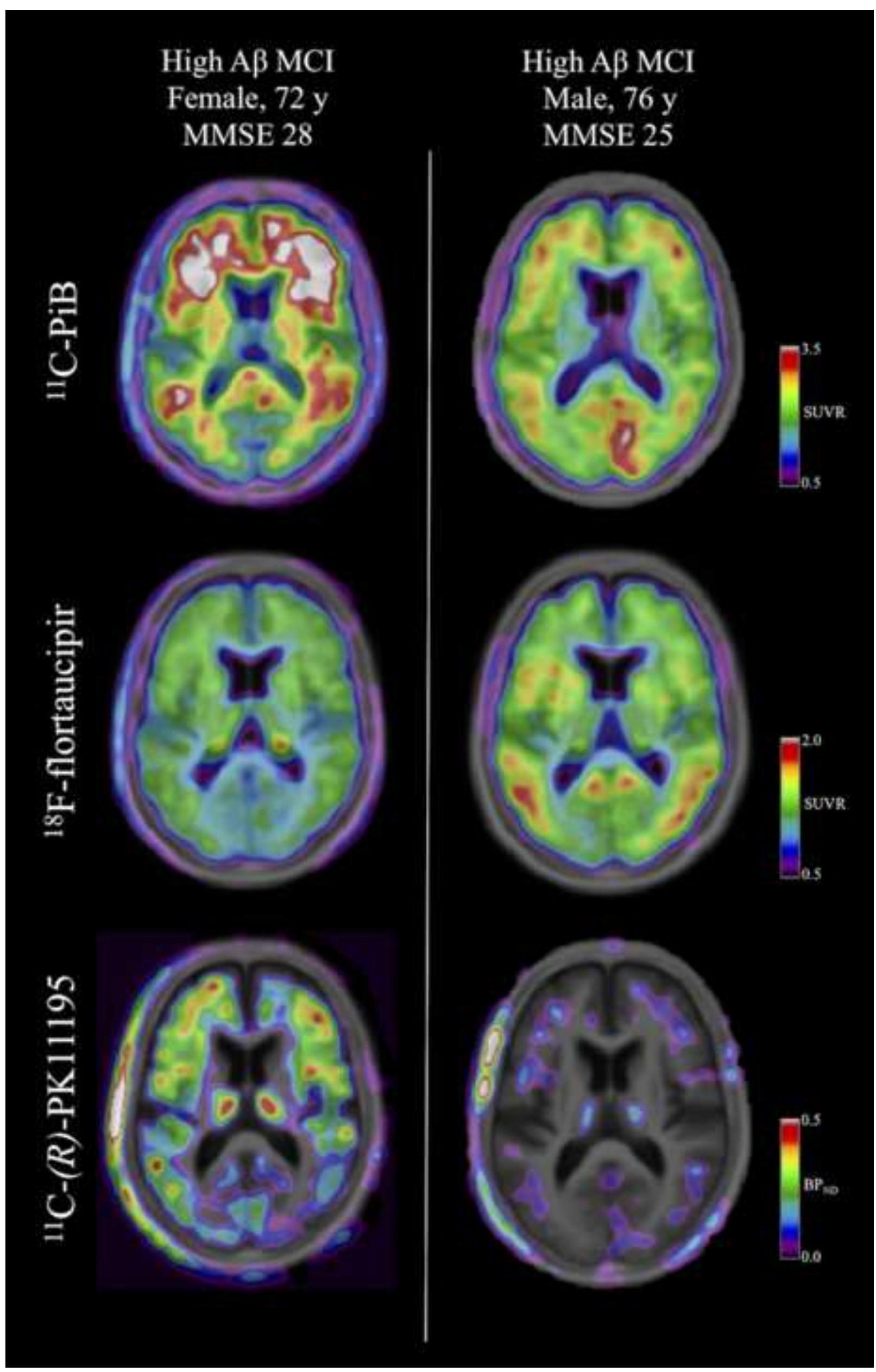

